\title{
Análisis del material didáctico para educación nutricional en pacientes con insuficiencia renal
}

\author{
Alejandro Molina Casco, Mercedes López-Pardo Martínez \\ Hospital Universitario Reina Sofía. Córdoba
}

\section{Sr. Director}

Para conseguir una correcta alimentación en la insuficiencia renal crónica terminal (IRCT), es imprescindible que el paciente aprenda a manejar los alimentos para obtener de ellos los nutrientes necesarios, en cantidad y proporción adecuadas según el estadio en que se encuentre la enfermedad. La información sanitaria sobre aspectos nutricionales juega un papel muy importante en los pacientes con IRCT, ya que la nutrición repercute directamente en el progreso de la enfermedad ${ }^{1}$.

Por tanto, la dieta en los pacientes con insuficiencia renal, debe ser cuidadosamente planificada, teniendo en cuenta que la información que reciban afectará a su salud de manera inmediata. Las fuentes más habituales por las que el paciente recibe información sanitaria son el material didáctico proporcionado en el hospital como apoyo en las consultas, material didáctico proporcionado por asociaciones de pacientes, e Internet.

El material educativo es un instrumento que se utiliza en educación para la salud, para facilitar la comunicación entre educador y paciente. Su finalidad es aportar los conocimientos suficientes, para crear hábitos saludables y para facilitar que el paciente asuma su estado de salud.

El material informativo tiene que ajustarse a los objetivos educativos de dicha intervención, y su finalidad será la de servir de apoyo para una correcta alimentación, la adherencia terapéutica y la observación de unas correctas medidas de hábitos saludables. Dentro del cuidado integral de enfermería al paciente con IRCT, es necesario dotar al paciente de los conocimientos

Correspondencia:

Alejandro Molina Casco

Avda. Las Ollerías 53. $2^{\circ} \mathrm{E} .14001$ Córdoba

E-mail: alejandro_casco_fr@hotmail.com necesarios sobre estos importantes aspectos del autocuidado, fundamentales para el proceso de evolución de su enfermedad, para conseguir la participación del paciente y su familia resultando esto imprescindible para garantizar el cumplimiento dietético².

El profesional enfermero deberá estar cualificado sobre los conocimientos dietéticos y saber evaluar el nivel que posee el paciente acerca de su dieta. Desde la primera visita en la consulta de enfermedad renal crónica avanzada se informará sobre los hábitos nutricionales y entregándole material didáctico.

Con las citas posteriores de seguimiento, se realizarán sesiones educativas solventando dudas sobre el material de apoyo impreso.

Un programa educativo dietético eficaz debe incluir información relativa a los siguientes aspectos:

- Objetivos de la dieta en la enfermedad renal.

- Aporte proteico y energético.

- Aporte de agua.

- Control de Na y K.

- Aporte vitamínico.

- Consejos dietéticos para controlar y disminuir los aportes de agua, $\mathrm{Na}$, K, P.

Y normas a seguir para la prevención de la hiperpotasemia y sobrecarga hídrica, y sobre cómo actuar ante la aparición de estas complicaciones.

Además, se incluirán ejemplos de menús y un listado de composición de alimentos, y consejos para la preparación de los mismos al objeto de reducir su contenido en potasio.

Estos contenidos deben estar dirigidos al paciente en un lenguaje sencillo y claro, para que la comunicación sea efectiva y el paciente comprenda la importancia de la dieta para preservar la función renal en su etapa prediálisis, y para evitar la desnutrición y la aparición de complicaciones una vez está en hemodiálisis ${ }^{3}$. 
Estudios previos ${ }^{2,3}$ demuestran que la comunicación enfermera-paciente es fundamental para lograr cambios en los hábitos del paciente. Por tanto, otro de los objetivos que debe cumplir un buen programa educativo es conseguir que el paciente sepa diferenciar la información de calidad que recibe en la consulta, y que se recoge en el material impreso que se le entrega, con otras fuentes de información que pueden encontrar en internet, las cuales pueden ser de dudosa fiabilidad.

En la valoración de la información sanitaria, existe un amplio debate sobre cuáles son los aspectos a cuidar para lograr una información de calidad.

Propuestas como la de $0^{\prime}$ Donnell ${ }^{4}$ que establecen los criterios que debe cumplir el material didáctico en materia de salud, o los tres criterios básicos que propone el Centre for Healt Information Quality de Gran Bretaña, se deben tener en cuenta a la hora de valorar la información sanitaria que el paciente recibe.

En la actualidad, el incremento en la demanda de información por parte del paciente por un lado, y el acceso ilimitado que tiene a multitud de fuentes a través de internet, están fomentando la aparición de instrumentos para la evaluación de la calidad de la información sanitaria que recibe el paciente, al ser fundamental una información de calidad en la toma de decisiones.

Un ejemplo es el cuestionario DISCERN (Anexo 1) que valora la fiabilidad de la publicación y la calidad de la información sobre los tratamientos sanitarios ${ }^{5}$.

Una revisión realizada por la Junta de Andalucía en $2004^{6}$ sobre las herramientas de ayuda a la decisión para pacientes concluye que aumentan el conocimiento en relación con su enfermedad y proporcionan un mayor realismo en las expectativas de los pacientes sobre los posibles efectos de una intervención. También reducen la pasividad en la toma de decisiones. Sin embargo sugiere que el desarrollo de estas iniciativas requiere impulsar mejoras de la comunicación con el paciente, incluyendo formación del profesional en habilidades, así como cambios organizativos y culturales.

Por otro lado, internet en la actualidad es una importante fuente de información sobre temas de salud. Dado que en la red existe mucha información de dudosa calidad o con criterios muy sesgados, están surgiendo diferentes entidades acreditadoras de calidad de la información, como la revisión y certificación HONcode (Anexo 2), que se utiliza para sitios web sanitarios para lograr la confiabilidad y utilidad de la información en Internet. El código HON solo verifica que el sitio se mantiene en los estándares, para que los lectores puedan saber la fuente y el propósito de la información médica presentada ${ }^{7}$.

Por tanto, nos planteamos como objetivo, analizar la calidad del material didáctico que reciben los usuarios con IRCT en la consulta del hospital y el que encuentran en plataformas Web, referentes a su dieta, durante el proceso de su enfermedad.

Para ello, hicimos una recogida de todo el material educativo referente a las recomendaciones para la nutrición del paciente renal en diferentes organismos y secciones del Hospital universitario Reina Sofía de Córdoba, y seleccionamos dos instrumentos de análisis de la calidad. DISCERN para el formato papel y HONcode para las páginas webs, ambos han sido traducidos para este trabajo.

Los criterios de inclusión para el material didáctico analizado por DISCERN fueron: su disponibilidad en centros sanitarios, encontrarse impresos, relación con el tema, material en idioma español y estar efectuados por profesionales sanitarios. Referente al material analizado por HONcode, los criterios de inclusión fueron: encontrarse en las 10 primeras páginas del motor de búsqueda de Google utilizando las palabras clave: dieta paciente renal.

Criterios de exclusión en DISCERN: artículos relacionados con medicinas alternativas y complementarias, no acceso a texto completo y artículos que no cumplían la metodología científica, empleando para ello la Guía CASPe de Lectura Crítica de la Literatura Médica, y textos en idioma distinto a inglés o español.

Criterios de exclusión en HONcode: webs con certificado de calidad o realizadas por un organismo oficial, páginas en inglés.

Se recopilaron los siguientes materiales educativos impresos:

"Guía para el Paciente Renal" de la planta de Diálisis del Hospital U. Reina Sofía, "Comer bien para vivir mejor teniendo Insuficiencia Renal", recogida del área de enfermería nutricional del Hospital Provincial de Córdoba, "Guía para el paciente renal" (Gobierno de España), "Guía de Alimentación en paciente con Insuficiencia Renal" (Hospital Donostia), "Alimentación saludable para personas en hemodiálisis" (American Kidney Fund) y la guía de ADAER de Murcia "Guía de Alimentación para pacientes renales". Este material se evaluó según los parámetros de calidad del cuestionario DISCERN. 
DISCERN consta de 15 preguntas clave, además de una calificación global de la calidad. Cada una de las 15 preguntas clave evalúa un criterio específico.

Las preguntas sobre DISCERN se organizan en tres secciones:

Preguntas 1-8 dirigidas a la fiabilidad de la publicación. Ayuda a considerar si se puede confiar en esa información como fuente fiable sobre las opciones de tratamiento

Pregunta 9 - 15 se dirigen a los detalles específicos de la información del tratamiento.

La pregunta 16 es la puntuación global de calidad. La respuesta a esta pregunta debe basarse en un juicio sobre la calidad de la publicación como fuente de información sobre las opciones de tratamiento, después de calificar cada una de las 15 preguntas anteriores.

Cada pregunta se califica en una escala de 5 puntos que va de No a Sí. La escala de calificación se ha diseñado para ayudarle a decidir si el criterio de la calidad en cuestión está presente o ha sido "cumplido" por la publicación. Las pautas generales son los siguientes:

- La puntuación 5 es un rotundo "sí" - el criterio de calidad se ha cumplido por completo.

- Parcialmente (2-4) si la publicación cumple el criterio de que se trata, en cierta medida.
- Una puntuación de 1 se debe dar si la respuesta a la pregunta es un rotundo "no" - el criterio de calidad no se ha cumplido en absoluto.

Por otro lado, se revisaron mediante el instrumento HONcode 11 páginas web.

Este instrumento, promovido por "Health On The Net Fundation", determina un conjunto de estándares de fiabilidad y credibilidad de la información disponible en los sitios web de salud, basado en 8 principios, que se muestran en el anexo 2, determinando la fiabilidad de las páginas web medida de $0-100 \%{ }^{7}$.

Este cuestionario hace referencia a los principios de autoridad, complementariedad, confidencialidad, atribución, justificabilidad, transparencia de la autoría, divulgación de información financiera y publicidad. Se cumplimenta registrando si la página Web examinada cumple o no con criterios relacionados con estos principios, dando como resultado el grado de fiabilidad de la página, calificándolas como webs muy seguras, seguras, o no seguras.

\section{Resultados}

El material didáctico en formato papel analizado con el instrumento DISCERN para determinar su calidad, obtuvo los resultados que se muestran en la tabla 1 .

Tabla 1. Puntuaciones de los criterios de calidad DISCERN y valoración global de calidad.

\begin{tabular}{|c|c|c|c|c|c|c|c|c|c|c|c|c|c|c|c|c|}
\hline Material didáctico & 1 & 2 & 3 & 4 & 5 & 6 & 7 & 8 & 9 & 10 & 11 & 12 & 13 & 14 & 15 & 16 \\
\hline $\begin{array}{l}\text { Guía para el paciente renal } \\
\text { según modalidades de } \\
\text { tratamiento. (Junta de } \\
\text { Andalucía) }\end{array}$ & 5 & 4 & 5 & 1 & 1 & 5 & 3 & 1 & 5 & 5 & 5 & 4 & 5 & 5 & 4 & $\begin{array}{c}\text { PARCIĀLMENTE: } \\
4\end{array}$ \\
\hline $\begin{array}{l}\text { ¿Qué puedo comer si tengo } \\
\text { insuficiencia renal? } \\
\text { (Abbott casa comercial) }\end{array}$ & 5 & 5 & 4 & 1 & 1 & 3 & 4 & 4 & 5 & 4 & 1 & 1 & 2 & 5 & 2 & $\begin{array}{l}\text { PARCIALMENTE: } \\
3\end{array}$ \\
\hline $\begin{array}{l}\text { Guía para el paciente renal } \\
\text { (Gobierno de España) }\end{array}$ & 5 & 5 & 5 & 4 & 5 & 4 & 3 & 1 & 5 & 5 & 4 & 3 & 5 & 4 & 4 & ALT0: 5 \\
\hline $\begin{array}{l}\text { Guía de Alimentación en } \\
\text { paciente con Insuficiencia } \\
\text { Renal ( Hospital Donostia) }\end{array}$ & 5 & 5 & 4 & 2 & 2 & 4 & 1 & 1 & 4 & 4 & 3 & 4 & 4 & 4 & 4 & $\begin{array}{c}\text { PARCIALMENTE: } \\
4\end{array}$ \\
\hline $\begin{array}{l}\text { Alimentación saludable } \\
\text { para personas en } \\
\text { hemodiálisis. (American } \\
\text { Kidney Fund) }\end{array}$ & 5 & 5 & 4 & 2 & 4 & 4 & 4 & 1 & 5 & 4 & 4 & 3 & 5 & 4 & 5 & $\begin{array}{c}\text { PARCIALMENTE: } \\
4\end{array}$ \\
\hline $\begin{array}{l}\text { Alimentación para } \\
\text { pacientes renales } \\
\text { (ADAER de Murcia) }\end{array}$ & 5 & 5 & 5 & 5 & 5 & 4 & 5 & 1 & 4 & 4 & 3 & 4 & 4 & 4 & 4 & ALTO: 5 \\
\hline
\end{tabular}


Con el instrumento HonCode para páginas web se valoraron 11 páginas webs.

En la Tabla 2, se muestran los grados de fiabilidad de las páginas valoradas con el instrumento HONcode.

Tabla 2: Grado de fiabilidad de Webs.

\begin{tabular}{|l|c|}
\hline Páginas web: & Fiabilidad \\
\hline www.webconsultas.com & $30 \%$ \\
\hline www.zonadiet.com & $20 \%$ \\
\hline www.ehowenespanol.com & $10 \%$ \\
\hline www.saludalia.com & $35 \%$ \\
\hline http://www.davidmsc.com & $5 \%$ \\
\hline http://www.equateq.com & $0 \%$ \\
\hline http://dietas-para-enfermedades.blogspot.com & $0 \%$ \\
\hline http://www.infirmus.es & $50 \%$ \\
\hline http://comobajardepesoymas.com & $25 \%$ \\
\hline http://www.innatia.com & $40 \%$ \\
\hline http://www.peybur.com & $10 \%$ \\
\hline
\end{tabular}

Nuestros resultados sugieren que sobre el material didáctico para la educación nutricional en el paciente con insuficiencia renal, existen unas diferencias en calidad y fiabilidad en función del tipo de fuente de información.

Los organismos oficiales como hospitales, organismos gubernamentales o las organizaciones muestran una información fiable que representa una ayuda para sus receptores, obteniendo el conocimiento necesario para el autocuidado en una de las necesidades básicas, según Virginia Henderson, como son la nutrición e hidratación, tal y como demuestran estudios como los de Sánchez Lamolda ${ }^{2}$ o Belchí Rosique ${ }^{3}$, donde se afirma que el aporte de material educativo adecuado para nutrición, tiene un balance positivo en la salud de los pacientes.

Teniendo en cuenta que la incidencia de la enfermedad renal va en aumento ${ }^{8}$, cifrándose actualmente en el $10 \%$ de la población adulta que sufre algún grado de IRCT, y que según estudios previos que determinan que las necesidades nutricionales del paciente renal alcanzan hasta el $42 \%$ de pacientes, se impone que por parte de los profesionales sanitarios se lleven a cabo programas de educación sanitaria en temas de nutrición, para cubrir esta necesidad del paciente.
En los últimos años, estamos asistiendo a un incremento en la utilización de fuentes de información científica en internet por parte del paciente, sin que esta información esté verificada por parte de profesionales sanitarios, siendo más que frecuente, y así lo hemos constatado en nuestro estudio, la aparición de páginas webs con informaciones no contrastadas, emitidas por personas no expertas o dirigidas a ciertos intereses distintos a la promoción de la salud, pudiendo originar serios problemas de salud a las personas que sigan sus consejos, todo ello agravado por el desconocimiento general sobre la existencia de instrumentos evaluadores de la fiabilidad de las página web.

Por otro lado, al evaluar el material impreso facilitado al paciente en la consulta de IRCT u otros centros sanitarios, encontramos que aportan al paciente información de calidad, segura, exacta y asequible, que además suele estar avalada por asociaciones científicas e incluso por organismos gubernamentales.

Internet es un lugar de búsqueda de información muy amplio en el que no hay controles para publicar informaciones que reúnan criterios mínimos de calidad.

Por tanto cualquier paciente que consulte sobre aspectos nutricionales, sin conocer cómo valorar la fiabilidad de la página que está consultando, puede correr riesgos al adquirir información sesgada o equívoca.

Por tanto podemos concluir que al ser de vital importancia para el paciente la calidad de la información que recibe en relación a su nutrición, al tener este tema una repercusión inmediata sobre su estado de salud, es imprescindible que los profesionales de la salud dirijan a los pacientes a fuentes fiables y seguras de información, facilitándole su acceso, y prevenir de esta forma la aparición de complicaciones por prácticas inadecuadas que deterioren su estado de salud.

\section{Bibliografía}

1. Rodolfo Crespo Montero, Rafael Casas Cuesta. Procedimientos y Protocolos con Competencias Específicas para Enfermería Nefrológica. $1^{\circ}$ ed. Madrid: Aulamédica; Octubre 2013.

2. Sánchez Lamolda MA, Fernández Herrerías G, Martínez Rodríguez AM, Malagón Rodríguez MA, Calatrava Fernández F. Intervención de Enfermería: Herramienta Clave para Mejorar el Estado Nutricional en los Pacientes con Insuficiencia Renal Crónica. Rev. Soc. Esp. Enferm. Nefrol. 2007; 10 (4): 287/291. 
3. F. Belchí Rosique, E. Merchán Mayado, C. Navarro Sánchez, M. Párraga Díaz, A. Rabadán Armero. Guía de alimentación para el paciente en hemodiálisis. Estudio descriptivo sobre su eficacia. Rev Soc Esp Enferm Nefrol 2001;4 (3) 12-19.

4. O'Donnell M, Entwistle V. Producing information about health and health care interventions: a practical guide. Aberdeen: Health Sciences Building, University of Aberdeen; 2003 [citado 10 Ago 2007]. Disponible en: http://www.abdn.ac.uk/ hsru/pdf/revisedguide_090603.pdf

5. Deborah Charnock, Sasha Shepperd, Gill Needham, Robert Gann. DISCERN: an instrument for judging the quality of written consumer health information on treatment choices. J Epidemiol Community Health 1999; 53:105-11.

6. Hermosilla-Gago, T y Briones, Ayuda a los usuarios en la toma de decisiones relacionadas con su salud.

Anexo 1. DISCERN

\section{$1^{a}$ Parte: ¿ES LA PUBLICACIÓN FIABLE?}

\section{I. ¿Está claro su tema principal?}

\begin{tabular}{l|l|l|l|l} 
No & Parcialmente & & & Sí \\
\hline 1 & 2 & 3 & 4 & 5
\end{tabular}

\section{SUGERENCIA}

Busque una indicación clara al comienzo de la publicación de qué se trata lo que está destinado a cubrir (y qué temas están excluidos).

Si la respuesta a la pregunta 1 es "No", pasar directamente a la pregunta 3.

\section{II. ¿Alcanza sus objetivos?}

\begin{tabular}{l|l|l|l|l} 
No & \multicolumn{2}{l|}{ Parcialmente } & Sí \\
\hline 1 & 2 & 3 & 4 & 5
\end{tabular}

\section{SUGERENCIA}

Considerar si la publicación contiene la información que apuntaba en la pregunta 1.

\section{III. ¿Es relevante?}

\begin{tabular}{l|l|l|l|l} 
No & \multicolumn{2}{|l|}{ Parcialmente } & Sí \\
\hline 1 & 2 & 3 & 4 & 5
\end{tabular}

\section{SUGERENCIA}

Considerar si: la publicación aborda las preguntas que los lectores podrían hacer Las recomendaciones y sugerencias sobre las opciones de tratamiento son realistas 0 apropiadas.
Herramientas disponibles y síntesis de la evidencia científica. Agencia de Evaluación de Tecnologías Sanitarias de Andalucía. España (2004). Sevilla.

7. Boyer C, Selby M, Appel RD, Scherrer JR. The Health on the Net Code of Conduct for Medical and Health Websites. Medinfo J Med Internet Res 1999; (1) supl 1: e99.

8. Otero A, de FA, Gayoso P, García F. Prevalence of chronic renal disease in Spain: results of the EPIRCE study. Nefrología 2010; 30 (1):78-86.

\section{IV. ¿Está claro qué fuentes de información se utilizaron para recopilar la publicación (además del autor o productor)?}

\begin{tabular}{l|l|l|l|l} 
No & \multicolumn{2}{l|}{ Parcialmente } & Sí \\
\hline 1 & 2 & 3 & 4 & 5
\end{tabular}

\section{SUGERENCIA}

Comprobar si los principales reclamos o declaraciones hechas sobre las opciones de tratamiento están acompañados por una referencia a las fuentes utilizadas como evidencia por ejemplo, un estudio de investigación u opinión experta.

Buscar un medio para comprobar las fuentes utilizadas, tales como una lista de bibliografía / referencia o las direcciones de los expertos y organizaciones citadas.

\section{V. ¿Está claro de dónde proviene la información usada en la publicación?}

\begin{tabular}{l|l|l|l|l} 
No & \multicolumn{2}{|l|}{ Parcialmente } & Sí \\
\hline 1 & 2 & 3 & 4 & 5
\end{tabular}

\section{SUGERENCIA}

Buscar las fechas de las principales fuentes de información utilizadas (pero no las fechas de reimpresión) Fecha de publicación (fecha de copyright). 
VI. ¿Es proporcionado o imparcial?

\begin{tabular}{l|l|l|l|l} 
No & \multicolumn{2}{|l|}{ Parcialmente } & Sí \\
\hline 1 & 2 & 3 & 4 & 5
\end{tabular}

SUGERENCIA

Busque una indicación clara de si la publicación está escrita desde un punto de vista objetivo o evidencias de que se utilizó una variedad de fuentes de información para elaborar la publicación, por ejemplo, más de un estudio de investigación o prueba pericial de la evaluación externa de la publicación.

La información no debe presentarse de una manera emocional 0 alarmista.

VII.¿Proporciona detalles de fuentes adicionales de apoyo e información?

\begin{tabular}{l|l|l|l|l} 
No & \multicolumn{2}{l|}{ Parcialmente } & Sí \\
\hline 1 & 2 & 3 & 4 & 5
\end{tabular}

\section{SUGERENCIA}

Buscar sugerencias de lecturas adicionales o para obtener detalles de otros organizaciones que presten asesoramiento e información sobre las condiciones y opciones de tratamiento.

\section{VIII.¿Hace referencia a áreas no fiables?}

\begin{tabular}{l|l|l|l|l} 
No & \multicolumn{2}{|l|}{ Parcialmente } & Sí \\
\hline 1 & 2 & 3 & 4 & 5
\end{tabular}

\section{SUGERENCIA}

Buscar las lagunas de conocimientos o las diferencias en la opinión de expertos relativa a las opciones de tratamiento.

Prestar atención si la publicación implica que una opción de tratamiento afecta a todos de la misma manera, por ejemplo, $100 \%$ de éxito con un tratamiento particular.

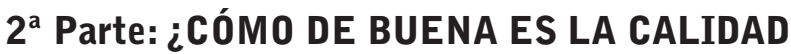 DE INFORMACIÓN SOBRE LAS OPCIONES DEL TRATAMIENTO?}

\section{IX.¿Describe cómo funciona cada tratamiento?}

\begin{tabular}{l|l|l|l|l} 
No & \multicolumn{2}{l|}{ Parcialmente } & Sí \\
\hline 1 & 2 & 3 & 4 & 5
\end{tabular}

\section{SUGERENCIA}

Buscar una descripción de cómo un tratamiento actúa sobre el cuerpo para lograr su efecto.

\section{X.¿¿Describe los beneficios de cada tratamiento?}

\begin{tabular}{l|l|l|l|l} 
No & \multicolumn{2}{|l|}{ Parcialmente } & Sí \\
\hline 1 & 2 & 3 & 4 & 5
\end{tabular}

\section{SUGERENCIA}

Los beneficios pueden incluir el control o la eliminación de los síntomas, la prevención de la recurrencia de la enfermedad y la eliminación de la enfermedad, tanto a corto plazo como a largo plazo.sobre el cuerpo para lograr su efecto.

\section{XI.¿Describe los riesgos de cada tratamiento?}

\begin{tabular}{l|l|l|l|l} 
No & \multicolumn{2}{|l|}{ Parcialmente } & Sí \\
\hline 1 & 2 & 3 & 4 & 5
\end{tabular}

\section{SUGERENCIA}

Los riesgos pueden incluir efectos secundarios, complicaciones y reacciones adversas al tratamiento, tanto a corto plazo como a largo plazo.

XII.¿Describe lo que ocurriría si no se utiliza ningún tratamiento?

\begin{tabular}{l|l|l|l|l} 
No & \multicolumn{2}{l|}{ Parcialmente } & Sí \\
\hline 1 & 2 & 3 & 4 & 5
\end{tabular}

\section{SUGERENCIA}

Buscar una descripción de los riesgos y beneficios del aplazamiento del tratamiento(es decir, el seguimiento de cómo avanza la enfermedad sin tratamiento).

XIII.¿Describe cómo las decisiones de tratamiento afectan la calidad de vida en general?

\begin{tabular}{l|l|l|l|l} 
No & \multicolumn{2}{|l|}{ Parcialmente } & Sí \\
\hline 1 & 2 & 3 & 4 & 5
\end{tabular}

\section{SUGERENCIA}

Buscar: descripción de los efectos de las opciones de tratamiento sobre la actividad diaria.

Descripción de los efectos de las opciones de tratamiento en las relaciones con la familia, amigos y cuidadores.

XIV.¿Está claro que puede haber más de una opción de tratamiento posible?

\begin{tabular}{l|l|l|l|l} 
No & \multicolumn{2}{l|}{ Parcialmente } & Sí \\
\hline 1 & 2 & 3 & 4 & 5
\end{tabular}

\section{SUGERENCIA}

Buscar lo siguiente: una descripción de quién tiene más probabilidades de beneficiarse de cada opción de tratamiento mencionado, y en qué circunstancias.

Sugerencias de alternativas a considerar o investigar más (incluyendo las opciones que no se describen completamente en la publicación) antes de decidir si se debe seleccionar o rechazar una opción de tratamiento particular.

XV.¿Proporciona soporte para la toma de decisiones compartida?

\begin{tabular}{l|l|l|l|l} 
No & \multicolumn{2}{|l|}{ Parcialmente } & Sí \\
\hline 1 & 2 & 3 & 4 & 5
\end{tabular}

\section{SUGERENCIA}

Buscar sugerencias de cosas que discutir con la familia, los amigos, los médicos u otros profesionales de la salud en relación con las opciones del tratamiento. 


\section{$3^{\text {a }}$ Parte: CLASIFICACIÓN GENERAL DE LA PUBLICACIÓN}

XVI. Con base en las respuestas a todas las preguntas anteriores, evaluar la calidad general de la publicación como fuente de información sobre las opciones de tratamiento.

\begin{tabular}{|c|c|c|c|c|}
\hline \multirow{2}{*}{$\begin{array}{l}\text { BAJ0: } \\
\text { Serios } \\
\text { defectos }\end{array}$} & \multicolumn{3}{|c|}{$\begin{array}{l}\text { MODERADO: potencialmente impor- } \\
\text { tante pero sin serios defectos }\end{array}$} & \multirow{2}{*}{$\begin{array}{l}\begin{array}{l}\text { ALTO: } \\
\text { Mínimos } \\
\text { defectos }\end{array} \\
5\end{array}$} \\
\hline & 2 & 3 & 4 & \\
\hline
\end{tabular}

Anexo 2. Certificación HONcode.

\section{Principio}

AUTORIDAD

La información disponible es realizada por profesionales en su totalidad.

\section{Principio}

\section{COMPLEMENTARIEDAD}

Información para apoyar, no reemplazar la relación médico-paciente.

\section{Principio}

CONFIDENCIALIDAD

Respetar la privacidad de los usuarios del sitio.

\section{Principio}

\section{ATRIBUCIÓN}

La información se respalda con a las fuentes de datos y con fecha de actualización.

\section{Principio}

\section{JUSTIFICABILIDAD}

Justificación de la información equilibrada y objetiva.

Principio

TRANSPARENCIA DE LA AUTORÍA.

Accesibilidad, proporcionar datos de contacto válidos para ampliar información sobre el contenido web.

Principio

DIVULGACIÓN DE INFORMACIÓN FINANCIERA

Proporciona detalles de financiación.

\section{Principio}

\section{PUBLICIDAD}

Distinguir claramente la publicidad del contenido editorial. 